books for the general public. The Nature of Space and Time has its origin in a series of lectures that they gave at a study programme in 1994 at the Isaac Newton Institute in Cambridge. They outline their past and current work on the global causal structure of space-time, singularity theorems, cosmic censorship, the laws of blackhole dynamics, quantum black holes, Schrödinger's cat paradox, the noboundary proposal, the arrow of time, and the twistor view of space-time. This is an extremely demanding book, with many equations, and requires some knowledge of general relativity and quantum theory. The main theme of the lectures is the quantization of general relativity. Hawking's work on quantum effects near black holes and Penrose's on twistor theory have proved enormously illuminating, but few physicists, I think, believe that a full quantization of general relativity will be achieved along these paths. A better bet, surely, is string theory, despite the difficulty of developing this to the state of testable predictions. The claims made for the noboundary proposal seem overstated. The 'prediction' of the microwave background fluctuations detected by COBE is a success also claimed by the whole generic class of inflationary theories. The debate between the positivist Hawking and the platonist Penrose on how the paradox of Schrödinger's cat should be understood is enjoyable, though the comparison with the classic debate between Einstein and Bohr in the 1920 s on the correct interpretation of quantum theory, made by Michael Atiyah in his introduction, seems a bit grandiose.

Donald Goldschmidt previously wrote a good book on Supernova 1987A, but Einstein's Greatest Blunder? is less successful. It is a rambling and shallow account of the present controversies about the Hubble constant and the nature of dark matter in the Universe, and whether the introduction of the cosmological constant (essentially an additional repulsive force acting on large scales permitted in general relativity) can resolve them. I was irritated by the approach implied by the book's subtitle: The Cosmological Constant and Other Fudge Factors in the Physics of the Universe. There are some surprising omissions, for example the results from the surveys of galaxy redshifts undertaken by IRAS. In the list of underground dark matter experiments, the UK experiment, currently setting the best limits, is ignored. Some of the figures are crude and inaccurate. You will learn far more about these issues from Silk's book.

There are not a great number of introductory textbooks on cosmology for undergraduates, but Marc Lachièze-Rey's Cosmology: A First Course is not a very distinguished addition to the list. There is a poor and inaccurate introductory chapter with some astrophysical background but, bizarrely, no mention of the microwave background radiation. The bulk of the book is a conventional account of the Friedmann models and their properties. The chapters on the early stages of the Big Bang and on galaxy formation give rather a limited physical insight. The greatest weakness is the virtual absence of discussion of observational cosmology. No index, references or list of further reading are provided.

Michael Rowan-Robinson is in the Astrophysics Group, Imperial College of Science, Technology and Medicine, Prince Consort Road, London SW7 2BZ, UK.

\section{Neural mysteries}

\section{Alison Abbott}

The Case of the Frozen Addicts. By J. William Langston and Jon Palfreman. Pantheon: 1995. Pp. 309. \$25.

"SWEDISH scientists use aborted fetuses to save drug addicts." This tabloid headline never existed, but the fear of it followed Bill Langston from the United States to Sweden where two of his patients underwent experimental surgery in 1989 to reverse their parkinsonism. This was a time when the world of neuroscience was gripped with excitement about a possible breakthrough in our understanding of Parkinson's disease. A chance discovery had opened up a new research direction in a field that for decades had stagnated, leading to an aggressive race to elaborate the biological mechanisms underlying the disease.

The discovery came not from a research bench but from the street, from the sad cases of six young Californian heroin addicts inadvertently exposed to a contaminated batch of a 'designer drug' in the summer of 1992. A few days after their bad shots, the addicts slowly froze. Completely unable to move or talk, the first victim, George Carillo, was presented to Langston, a local neurologist, who recognized the symptoms of severe parkinsonism, a degenerative motor disease normally confined to the elderly. Carillo had already been misdiagnosed by several doctors, all convinced he was malingering to avoid a prison sentence.

At the time, Langston was a clinical neurologist in a mundane county medical centre in California. He had ambitions to do basic research, but had neither the resources nor the training. The addicts proved to be his professional saving. The Case of the Frozen Addicts tells the tale of how Langston found his fortune in worlds initially foreign to him: the grim reality of drug addiction in America's underclass; the aggressive competitiveness of medical research; the devious politics of the
National Institutes of Health (NIH); and the ultra-conservative politics of the Bush administration. Langston, and the other bit-part actors in the story, had to confront all of these in solving the mystery of the frozen addicts and applying the knowledge to advance our understanding of Parkinson's disease and other disorders of voluntary movement.

It is a tale of several parallel strands. We learn how the police eventually tracked down the manufacturer of the contaminated drug (he was formerly a public-spirited lawyer); how sample analysis revealed the toxic contaminant to be MPTP; and how Langston and other scientists came to discover that MPTP, after metabolic conversion to $\mathrm{MPP}^{+}$, selectively destroys the dopamine-producing cells in the substantia nigra, a region of the brain involved in the control of movement. It was this finding that led to the first animal model of Parkinson's disease and so to an explosion of basic research into the disease.

The authors explain both the detective story and the science through the eyes of the personalities involved. One feels for Langston both when he suffers the practical consequences of professional jealousy and when he falls foul of the NIH, which 'poached' one of the addicts behind his back. (The moral here is that a small-time scientist cannot take on the might of the NIH.) Against all the odds, however, Langston managed to establish his own private treatment and research centre. We also gain insight into the minds of drug addicts, as well as sympathize with their agony, and that of their families, on finding themselves faced with a hellish choice: to remain trapped in an immobile body or to suffer the severe side-effects of the only medication, L-dopa, that could free their limbs.

The story has a happy ending. Three of the addicts have benefited from a controversial new technique, pioneered in Sweden, of transplantation of substantia nigra cells from human fetuses into the brains of parkinsonism sufferers. The use of fetal tissue in medicine was outlawed in the US just as the first scientists applied for permission to attempt such transplants at the NIH in 1988. (The ban was lifted in 1991.)

Although the authors provide an accurate and accessible description of the science, they do occasionally show a regrettable lack of judgement in giving equally enthusiastic weight to all theories, including the doubtful proposal that an environmental toxin, with a similar mechanism to MPTP, may be a prime cause of 'normal' parkinsonism. Nevertheless, the book succeeds in bringing science to life and showing its relevance to human health. It also illuminates the complexities that can surround researchers less isolated from the real world than they might otherwise imagine.

Alison Abbott is Nature's Senior European Correspondent. 